\title{
Overexpression of the Transmembrane Protein IQGAP3 Is Associated with Poor Survival of Patients with Gastric Cancer
}

\author{
Naohide Oue ${ }^{a}$ Yuji Yamamoto $^{a}$ Takashi Oshimab $^{b}$ Ryuichi Asai ${ }^{a}$

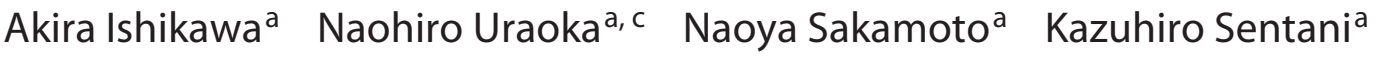 \\ Wataru Yasui ${ }^{a}$ \\ ${ }^{a}$ Department of Molecular Pathology, Hiroshima University Institute of Biomedical and Health Sciences, \\ Hiroshima, Japan; ${ }^{b}$ Department of Surgery, Yokohama City University, Yokohama, Japan; ${ }^{C}$ Department of \\ Translational Molecular Pathology, The University of Texas MD Anderson Cancer Center, Houston, TX, USA
}

\author{
Keywords \\ IQGAP3 · Mucin phenotype · Spheroid · Cancer stem cell · \\ Gastric cancer
}

\begin{abstract}
Objective: Spheroid colony formation is a useful method of cancer stem cell (CSC) characterization. We previously showed that the IQ motif containing the GTPase-activating protein 3 gene (IQGAP3) is upregulated in spheroid bodyforming gastric cancer (GC) cells compared with parental cells. We investigated IQGAP3 expression in GC. Methods: IQGAP3 protein expression was analyzed by immunohistochemistry in 165 GC cases. RNA interference was used to inhibit IQGAP3 expression in GC cell lines. Results: In nonneoplastic gastric mucosa, weak staining of IQGAP3 was observed in the foveolar epithelium, while GC tissue showed stronger, more extensive staining. Of the 165 GC cases, 34 (21\%) were positive for IQGAP3 expression. GC cases positive for IQGAP3 were found more frequently in stage II/III/IV cases than in stage I cases. Univariate and multivariate analyses demonstrated that IQGAP3 expression is an independent prognostic classifier of GC patients. Both the number and size
\end{abstract}

of the spheres formed by MKN-74 cells were significantly reduced by knockdown of IQGAP3. The phosphorylation of Akt and Erk1/2 was inhibited by knockdown of IQGAP3. Conclusion: These results suggest that IQGAP3 plays an important role in gastric CSCs. The location of IQGAP3 on the cell membrane makes it a potential therapeutic target for GC.

(c) 2017 S. Karger AG, Basel

\section{Introduction}

Gastric cancer (GC) is one of the most common human cancers. Multiple genetic and epigenetic alterations are associated with GC, so enhanced knowledge of the gene expression changes that occur during gastric carcinogenesis may facilitate improvements in disease diagnosis, treatment, and prevention [1]. Genes encoding transmembrane/secretory proteins that are expressed specifically in cancers are ideal diagnostic biomarkers. Moreover, if the products of these genes function in the neoplastic process, they may also be suitable therapeutic targets [2].

During the past decade, cancer has been recognized as a stem cell disease [3], and cancer stem cells (CSCs) have

\section{KARGER}

(C) 2017 S. Karger AG, Basel

E-Mail karger@karger.com

www.karger.com/pat
Naohide Oue

Department of Molecular Pathology

Hiroshima University Institute of Biomedical and Health Sciences

1-2-3 Kasumi, Minami-ku, Hiroshima 734-8551 (Japan)

E-Mail naoue@ hiroshima-u.ac.jp 
been described in numerous solid tumors. Gastric CSCs are characterized by the expression of specific cell surface markers including CD44, CD133, and aldehyde dehydrogenase 1 (ALDH1) [4]. However, gastric CSCs have not been completely characterized.

Spheroid colony formation is a useful method with which to characterize gastric CSCs [5]. Previously, we reported on the upregulation of genes in spheroid bodyforming GC cell lines compared with parental cells [6]. We showed that KIFC1 and KIF11 protein expression is upregulated in 37 and $72 \%$, respectively, of GC cases, and that both the number and size of spheres from GC cell lines are significantly reduced in KIFC1 and KIF11 small interfering (si)RNA-transfected cells compared with negative control siRNA-transfected cells $[6,7]$. However, the expression of many genes remains unconfirmed, and their role in GC is unclear.

In the present study, we focused on the IQ motif containing the GTPase-activating protein 3 gene (IQGAP3), because we previously observed it to be upregulated in spheroid body-forming cells compared with parental cells [6]. Moreover, because IQGAP3 is a transmembrane protein, it has the potential to be a therapeutic target [8]. The IQGAP family comprises 3 members, IQGAP1, IQGAP2, and IQGAP3 [9], which all contain 4 IQ motifs and a RasGAP-related domain [10]. IQGAP1 has been suggested to function in cell migration and regulation of the cytoskeleton [11], and it may play a role in cancer progression [12]. In contrast, IQGAP2 seems to act as a tumor suppressor [13], while IQGAP3 is thought to be involved in the proliferation of epithelial cells [14]. However, the role of IQGAP3 in GC pathogenesis remains unclear.

Herein, we analyzed the expression and distribution of IQGAP3 protein in human GC using immunohistochemistry, and examined the relationship between IQGAP3 expression and clinicopathologic characteristics. Furthermore, because gastric and intestinal mucin phenotypes of GC have distinct clinical characteristics and exhibit specific genetic and epigenetic changes [1], associations between IQGAP3 expression and gastric/intestinal mucin phenotypes were investigated. We also analyzed the effect of inhibiting IQGAP3 expression by RNA interference (RNAi) on spheroid formation by GC cells.

\section{Materials and Methods}

\section{Tissue Samples}

In a retrospective study, samples from 175 primary tumors were collected from patients diagnosed with GC who underwent surgery between 2003 and 2008 at Hiroshima University Hospital
(Hiroshima, Japan). This study was approved by the Ethics Committee for Human Genome Research of Hiroshima University (Hiroshima, Japan). All patients underwent curative resection. Only patients without preoperative radiotherapy or chemotherapy and without clinical evidence of distant metastasis were enrolled in the study. Operative mortality was defined as death within 30 days of leaving hospital, and these patients were removed from the analysis. Postoperative follow-up was scheduled every 1, 2, or 3 months during the first 2 years after surgery and every 6 months thereafter unless more frequent follow-up was deemed necessary. Chest Xray, chest computed tomography scan, and serum chemistries were performed at every follow-up visit. Patients were followed by their physicians until their death or the date of the last documented contact.

For quantitative reverse transcription (qRT)-PCR, 10 GC samples were used. Samples were frozen immediately in liquid nitrogen and stored at $-80^{\circ} \mathrm{C}$ until use. For immunohistochemical analysis, archival formalin-fixed, paraffin-embedded tissues from 165 patients who had undergone surgical excision for GC were used. One or two representative tumor blocks, including the tumor center, invading front, and tumor-associated nonneoplastic mucosa, from each patient were examined using immunohistochemistry. In cases of large, late-stage tumors, 2 different sections were examined to include representative areas of the tumor center as well as of the lateral and deep tumor invasive fronts. Tumor staging was determined according to the tumor-node-metastasis (TNM) classification system [15].

\section{qRT-PCR Analysis}

Total RNA was extracted from GC samples using an RNeasy Mini Kit (Qiagen, Valencia, CA, USA), and $1 \mu \mathrm{g}$ of total RNA was converted to cDNA using the First Strand cDNA Synthesis Kit (Amersham Biosciences, Piscataway, NJ, USA). Quantitation of IQGAP3 mRNA levels was performed by real-time fluorescence detection as described previously [16]. PCR was conducted using the SYBR Green PCR Core Reagents Kit (Applied Biosystems; Thermo Fisher Scientific, Inc., Waltham, MA, USA). Real-time detection of the emission intensity of SYBR Green bound to double-stranded DNA was performed with the ABI PRISM 7700 Sequence Detection System (Applied Biosystems; Thermo Fisher Scientific, Inc.). ACTB-specific PCR products were amplified from the same RNA samples and served as an internal control.

\section{Immunohistochemistry}

Immunohistochemical analysis was performed with a Dako Envision+ Mouse/Rabbit Peroxidase Detection System (Dako Cytomation, Carpinteria, CA, USA). Antigen retrieval was performed by microwave heating in citrate buffer $(\mathrm{pH} \mathrm{6.0)}$ for $30 \mathrm{~min}$. Peroxidase activity was blocked with $3 \% \mathrm{H}_{2} \mathrm{O}_{2}$-methanol for $10 \mathrm{~min}$, and sections were incubated with normal goat serum (Dako Cytomation) for 20 min to block nonspecific antibody binding sites. Sections were incubated with a rabbit polyclonal anti-IQGAP3 antibody (1:50, kindly provided by Prof. Sachiko Tsukita, Osaka University, Osaka, Japan) for $1 \mathrm{~h}$ at room temperature, followed by incubation with Envision+ anti-rabbit peroxidase for $1 \mathrm{~h}$. For color reactions, sections were incubated with the DAB SubstrateChromogen Solution (Dako Cytomation) for $10 \mathrm{~min}$. Sections were counterstained with $0.1 \%$ hematoxylin. Reactions lacking a primary antibody were used as negative controls. 


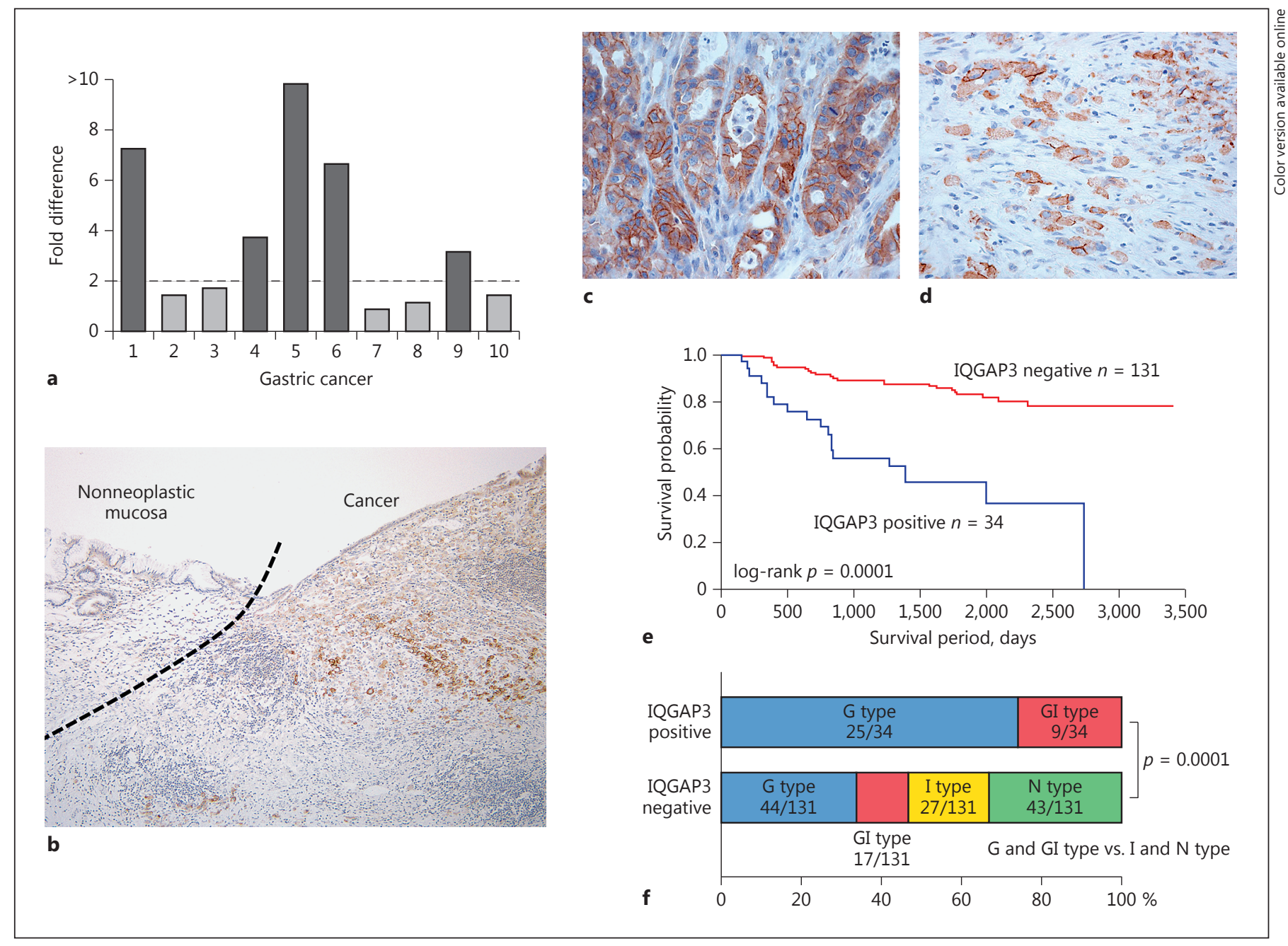

Fig. 1. Expression of IQGAP3 in gastric cancer (GC). a qRT-PCR analysis of IQGAP3 in $10 \mathrm{GC}$ samples. The bars represent individual samples. Fold-change indicates the ratio of IQGAP3 mRNA expression in GC to that in corresponding nonneoplastic mucosa. b Immunohistochemical analysis of IQGAP3 in GC and corresponding nonneoplastic gastric mucosa. Original magnification, $\times 40$. c Immunohistochemical analysis of IQGAP3 in well-differentiated type GC. Original magnification, $\times 400$. d Immunohistochemical analysis of IQGAP3 in poorly-differentiated type GC. Original magnification, $\times 400$. e Kaplan-Meier plot of survival in patients with GC. $\mathbf{f}$ Summary of IQGAP3 expression and expression of the mucin phenotype.
The expression of IQGAP3 was scored in all tumors as positive or negative. When more than $10 \%$ of tumor cells were stained, immunostaining was considered positive for IQGAP3. Using these definitions, two surgical pathologists (N.O. and K.S.) without knowledge of clinical and pathologic parameters or patient outcome independently reviewed immunoreactivity in each specimen. Interobserver differences were resolved by consensus review at a double-headed microscope after the independent review.

\section{Phenotypic Analysis}

GCs were classified into 4 phenotypes: gastric (G) type, intestinal (I) type, gastric and intestinal mixed (GI) type, and null (N) type. For phenotypic expression analysis of GC, we performed immunohistochemical analysis (as described above) with 4 antibodies: anti-MU-
C5AC (Novocastra, Newcastle, UK) as a marker of foveolar epithelial cells in the stomach, anti-MUC6 (Novocastra) as a marker of pyloric gland cells in the stomach, anti-MUC2 (Novocastra) as a marker of goblet cells in the small intestine and colorectum, and anti-CD10 (Novocastra) as a marker of microvilli of absorptive cells in the small intestine and colorectum. The criteria for the classification of G type and I type GCs have been described previously [1]. Briefly, GCs in which more than $10 \%$ of cells in the section expressed at least one gastric epithelial cell marker (MUC5AC or MUC6) or intestinal epithelial cell marker (MUC2 or CD10) were classified as $\mathrm{G}$ type or I type cancers, respectively. Sections that showed both gastric and intestinal phenotypes were classified as GI type, and those that lacked both the gastric and intestinal phenotypes were classified as $\mathrm{N}$ type. The phenotype status has been determined previously [7]. 


\section{Cell Lines}

Four cell lines derived from human GC (MKN-1, MKN-7, $\mathrm{MKN}-45$, and MKN-74) were purchased from the Japanese Collection of Research Bioresources Cell Bank (Osaka, Japan). All cell lines were maintained in RPMI 1640 (Nissui Pharmaceutical Co., Ltd., Tokyo, Japan) containing 10\% fetal bovine serum (BioWhittaker; Lonza, Basel, Switzerland) in a humidified atmosphere of $5 \%$ $\mathrm{CO}_{2}$ and $95 \%$ air at $37^{\circ} \mathrm{C}$.

\section{RNA Interference}

siRNA oligonucleotides targeting IQGAP3 and a negative control were purchased from Invitrogen (Thermo Fisher Scientific, Inc.). We used 2 independent IQGAP3 siRNA oligonucleotide sequences. Transfection was performed using Lipofectamine RNAiMAX (Invitrogen) as described previously [17]. Briefly, 60 pmol of siRNA and $10 \mu \mathrm{L}$ of Lipofectamine RNAiMAX were mixed in $1 \mathrm{~mL}$ of RMPI medium ( $10 \mathrm{nmol} / \mathrm{L}$ final siRNA concentration). After 20 min of incubation, the mixture was added to cells, which were then plated in culture dishes. Cells were analyzed $48 \mathrm{~h}$ after transfection.

\section{Western Blot Analysis}

Cells were lysed as described previously [18]. Lysates (40 $\mu \mathrm{g})$ were solubilized in Laemmli sample buffer by boiling and then subjected to $10 \%$ sodium dodecyl sulfate polyacrylamide gel electrophoresis followed by electrotransfer onto nitrocellulose membranes. Anti-extracellular signal-regulated kinase 1/2 (Erk1/2), anti-phospho-Erk1/2 (pErk1/2), anti-Akt, and anti-phospho-Akt (pAkt) were purchased from Cell Signaling Technology (Danvers, MA, USA). Peroxidase-conjugated anti-mouse or anti-rabbit IgG was used in the secondary reaction. Immunocomplexes were visualized with an ECL Western Blot Detection System (Amersham Biosciences). $\beta$-Actin (AC-15; Sigma Chemical, St. Louis, MO, USA) was also stained as a loading control.

\section{Cell Growth Assay}

To examine cell growth, we performed 3-(4,5-dimethylthiazol2-yl)-2,5-diphenyltetrazolium bromide (MTT) assays. The cells were seeded at a density of 2,000 cells per well in 96-well plates. Cell growth was examined after 1, 2, and 4 days. Three independent experiments were performed, and means and standard deviations (SD) were calculated for each experiment.

\section{Spheroid Colony Formation}

For the generation of spheres, 2,000 cells were plated per well in 24-well ultra-low attachment plates (Corning; New York, NY, USA). Cells were grown in mTeSR medium (STEMCELL Technologies Inc., Cambridge, MA, USA). The plates were incubated at $37^{\circ} \mathrm{C}$ in a $5 \% \mathrm{CO}_{2}$ incubator for 15 days, after which the sphere number and size were determined under a microscope.

\section{Statistical Analysis}

Associations between clinicopathologic parameters and IQGAP3 expression were analyzed by the Fisher exact test. Kaplan-Meier survival curves were constructed for IQGAP3-positive and IQGAP3-negative patients. Survival rates were compared between IQGAP3-positive and IQGAP3-negative groups. Differences between survival curves were tested for statistical significance by a log-rank test. Differences in the sphere number and size between the 2 groups were tested by the Student $t$ test.

IQGAP3 Expression in Gastric Cancer
Table 1. Relationships between IQGAP3 expression and clinicopathologic characteristics

\begin{tabular}{|c|c|c|c|}
\hline & \multicolumn{2}{|c|}{ IQGAP3 expression } & \multirow[t]{2}{*}{$p$ value } \\
\hline & positive & negative & \\
\hline \multicolumn{4}{|l|}{ Age } \\
\hline$\leq 65$ years & $19(23 \%)$ & 65 & \multirow[t]{2}{*}{0.5668} \\
\hline$>65$ years & $15(19 \%)$ & 66 & \\
\hline \multicolumn{4}{|l|}{ Sex } \\
\hline Male & $21(21 \%)$ & 81 & \multirow[t]{2}{*}{1.0000} \\
\hline Female & $13(21 \%)$ & 50 & \\
\hline \multicolumn{4}{|l|}{ T classification } \\
\hline $\mathrm{T} 1$ & $10(14 \%)$ & 62 & \multirow[t]{2}{*}{0.0783} \\
\hline $\mathrm{T} 2 / 3 / 4$ & $24(26 \%)$ & 69 & \\
\hline \multicolumn{4}{|l|}{$\mathrm{N}$ classification } \\
\hline N0 & $11(13 \%)$ & 74 & \multirow[t]{2}{*}{0.0132} \\
\hline $\mathrm{N} 1 / 2 / 3$ & $23(29 \%)$ & 57 & \\
\hline \multicolumn{4}{|l|}{ M classification } \\
\hline M0 & $22(17 \%)$ & 111 & \multirow[t]{2}{*}{0.0139} \\
\hline M1 & $12(38 \%)$ & 20 & \\
\hline \multicolumn{4}{|l|}{ Stage } \\
\hline Stage I & $10(12 \%)$ & 72 & \multirow[t]{2}{*}{0.0117} \\
\hline Stage II/III/IV & $24(29 \%)$ & 59 & \\
\hline \multicolumn{4}{|l|}{ Histologic classification } \\
\hline Well differentiated & $16(20 \%)$ & 65 & \multirow[t]{2}{*}{0.8487} \\
\hline Poorly differentiated & $18(21 \%)$ & 66 & \\
\hline \multicolumn{4}{|l|}{ Lymphatic invasion } \\
\hline Positive & $23(25 \%)$ & 70 & \multirow[t]{2}{*}{0.1748} \\
\hline Negative & $11(15 \%)$ & 61 & \\
\hline \multicolumn{4}{|l|}{ Vascular invasion } \\
\hline Positive & $18(25 \%)$ & 54 & \multirow[t]{2}{*}{0.2476} \\
\hline Negative & $16(17 \%)$ & 77 & \\
\hline
\end{tabular}

\section{Results}

\section{Expression of IQGAP3 in GC}

We previously showed that IQGAP3 expression was twofold higher in spheroid body-forming GC cells compared with parental cells. Here, we first analyzed IQGAP3 mRNA expression in 10 GC tissue samples and nonneoplastic mucosa samples by qRT-PCR (Fig. 1a), and observed the overexpression of IQGAP3 (tumor/nonneoplastic mucosa ratio $>2)$ in $5(50 \%)$ of 10 GC tissue samples.

Next, we performed immunohistochemical analysis of IQGAP3 in 165 GC tissue samples. In nonneoplastic gastric mucosa, weak staining of IQGAP3 was observed in the foveolar epithelium. No staining of IQGAP3 was found in stromal cells. In contrast, GC tissue showed stronger, more extensive staining (Fig. 1b) in both welldifferentiated GC (Fig. 1c) and poorly-differentiated GC (Fig. 1d). A total of 34 (21\%) GC cases were positive for 
Table 2. Univariate and multivariate Cox regression analysis of IQGAP3 expression and survival in gastric cancer

\begin{tabular}{|c|c|c|c|c|}
\hline \multirow[t]{2}{*}{ Characteristic } & \multicolumn{2}{|l|}{ Univariate analysis } & \multicolumn{2}{|l|}{ Multivariate analysis } \\
\hline & HR (95\% CI) & $p$ value & $\operatorname{HR}(95 \%$ CI) & $p$ value \\
\hline \multicolumn{5}{|l|}{ Age } \\
\hline$\leq 65$ years & 1 (Ref.) & & & \\
\hline$>65$ years & $1.12(0.61-2.04)$ & 0.7188 & & \\
\hline \multicolumn{5}{|l|}{ Sex } \\
\hline Male & 1 (Ref.) & & & \\
\hline Female & $1.52(0.81-3.02)$ & 0.1945 & & \\
\hline \multicolumn{5}{|l|}{ Stage } \\
\hline I & 1 (Ref.) & & 1 (Ref.) & \\
\hline II/III/IV & $28.40(8.72-174.37)$ & 0.0001 & $20.48(4.89-142.24)$ & 0.0001 \\
\hline \multicolumn{5}{|l|}{ Histologic classification } \\
\hline Well differentiated & 1 (Ref.) & & & \\
\hline Poorly differentiated & $1.51(0.83-2.81)$ & 0.1817 & & \\
\hline \multicolumn{5}{|l|}{ Lymphatic invasion } \\
\hline Negative & 1 (Ref.) & & 1 (Ref.) & \\
\hline Positive & $9.57(3.85-31.92)$ & 0.0001 & $1.62(0.52-6.50)$ & 0.4289 \\
\hline \multicolumn{5}{|l|}{ Vascular invasion } \\
\hline Negative & 1 (Ref.) & & 1 (Ref.) & \\
\hline Positive & $3.63(1.93-7.20)$ & 0.0001 & $0.64(0.42-1.81)$ & 0.6392 \\
\hline \multicolumn{5}{|l|}{ IQGAP3 expression } \\
\hline Negative & 1 (Ref.) & & 1 (Ref.) & \\
\hline Positive & $4.60(2.47-8.44)$ & 0.0001 & $3.49(1.89-6.46)$ & 0.0002 \\
\hline
\end{tabular}

IQGAP3. IQGAP3 staining was mainly observed on the cell membrane. Many GC cases exhibited IQGAP3 staining heterogeneity, with the percentage of IQGAP3stained GC cells ranging from 0 to $70 \%$. No tendency for IQGAP3 upregulation at the invasive front was observed.

We next examined the relationship between IQGAP3 staining and clinicopathologic characteristics (Table 1). A marginally significant difference was observed between IQGAP3 expression and T classification $(p=0.0783)$. In contrast, IQGAP3 expression was statistically associated with $\mathrm{N}$ classification $(p=0.0132)$ and $\mathrm{M}$ classification ( $p=0.0139)$. GC cases positive for IQGAP3 were found more frequently in stage II/III/IV cases than in stage I cases. These results suggest that IQGAP3 plays an important role in the progression of GC. Furthermore, KaplanMeier analysis demonstrated that IQGAP3-positive GC cases had significantly worse survival than IQGAP3-negative GC cases ( $p=0.0001$; Fig. 1e). We next performed univariate and multivariate Cox proportional hazard analyses to evaluate the potential use of IQGAP3 expression as a prognostic classifier (Table 2). In univariate analysis, stage, lymphatic invasion, vascular invasion, and IQGAP3 expression were associated with poor survival.
Table 3. Relationships between IQGAP3 expression and gastric/ intestinal phenotype markers

\begin{tabular}{lccc}
\hline & \multicolumn{2}{c}{ IQGAP3 expression } & \multirow{2}{*}{$p$ value } \\
\cline { 2 - 3 } & positive & negative & \\
\hline MUC5AC & & & \\
$\quad$ Positive & $30(34 \%)$ & 57 & 0.0001 \\
Negative & $4(5 \%)$ & 74 & 0.0422 \\
MUC6 & $7(39 \%)$ & 11 & \\
$\quad$ Positive & $27(18 \%)$ & 120 & 0.8417 \\
Negative & $8(20 \%)$ & 33 & \\
MUC2 & $26(21 \%)$ & 98 & 0.0944 \\
$\quad$ Positive & $1(6 \%)$ & 22 & \\
Negative & $33(22 \%)$ & 114 & \\
CD10 & & & \\
$\quad$ Positive & & & \\
Negative & & & \\
\hline
\end{tabular}

In the multivariate model, stage and IQGAP3 expression were found to be independent predictors of survival in patients with GC. These results suggest that IQGAP3 is a promising biomarker with which to identify patients with poor prognosis. 
Fig. 2. Effect of IQGAP3 knockdown in gastric cancer (GC) cells. a Western blot analysis of IQGAP3 protein expression in 4 GC cell lines. b Western blot analysis of IQGAP3, Akt, phospho-Akt (pAkt), Erk1/2, and phospho-Erk1/2 (pErk1/2) in cell lysates from MKN-74 cells transfected with IQGAP3 siRNA or negative control siRNA. $\beta$-Actin was measured as a loading control. c Cell growth was assessed by MTT assays at days 1,2, and 4 after seeding in 96-well plates. Bars and error bars indicate the mean and SD, respectively, of 3 independent experiments. ${ }^{\star} p<0.05$. d The number of spheres from MKN-74 cells transfected with IQGAP3 siRNA or negative control siRNA. Bars and error bars indicate the mean and SD, respectively, of 3 independent experiments. ${ }^{\star} p<0.05$. e The size of spheres from MKN-74 cells transfected with IQGAP3 siRNA or negative control siRNA. Bars and error bars indicate the mean and SD, respectively, of 3 independent experiments. ${ }^{\star} p<0.05$.

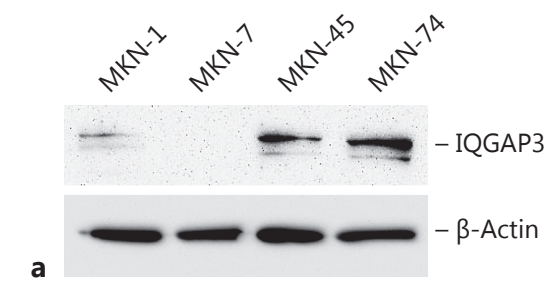

a
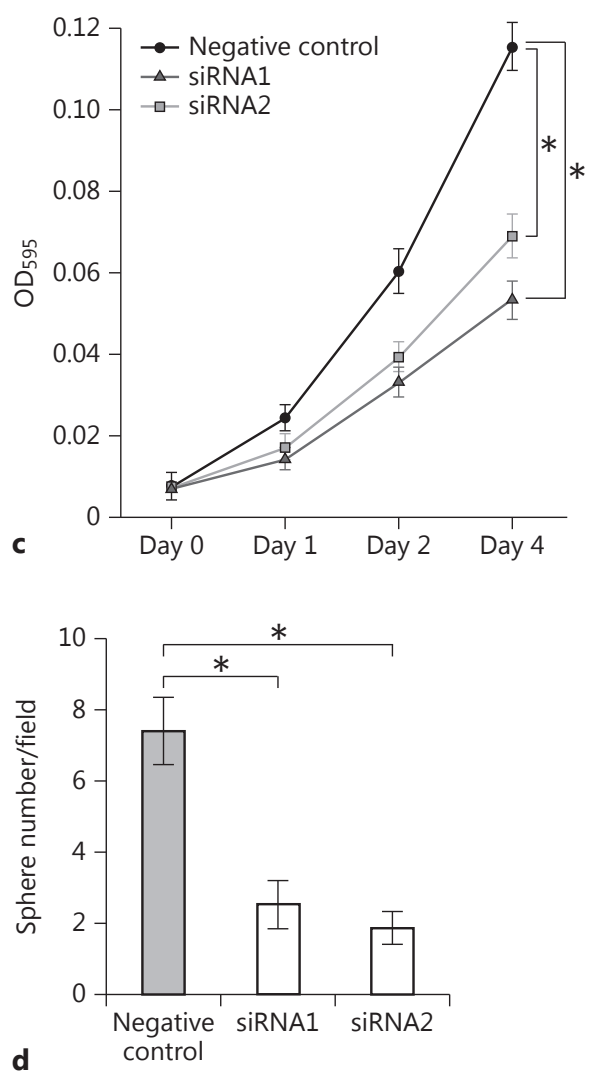
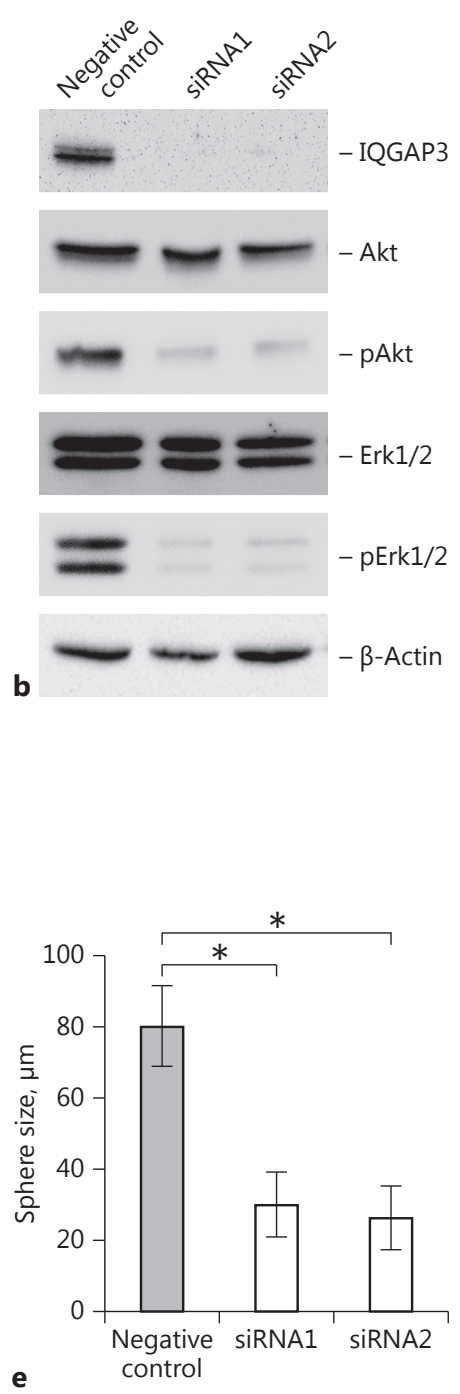

Association between IQGAP3 Expression and Gastric/ Intestinal Phenotype Marker

We investigated associations between IQGAP3 expression and the mucin phenotype (G type, I type, GI type, and $\mathrm{N}$ type). Immunohistochemical analysis of gastric (MUC5AC and MUC6) and intestinal (MUC2 and CD10) markers was used to phenotypically classify the $165 \mathrm{GC}$ cases as $\mathrm{G}$ type $(n=69,42 \%)$, I type $(n=27,16 \%)$, GI type $(n=26,16 \%)$, and $\mathrm{N}$ type $(n=43,26 \%)$. IQGAP3 was shown to be frequently expressed in MUC5AC-positive and MUC6-positive GC cases (Table 3), and was ob- served significantly more frequently in GC exhibiting the G phenotype ( $G$ type GC plus GI type GC) than in other GC types (I type GC plus N type GC) $(p=0.0001$, Fisher exact test; Fig. 1f).

\section{Effect of Inhibition of IQGAP3 on Sphere Number and} Size

Our results showed that IQGAP3 was frequently expressed in GC cases, and GC cases positive for IQGAP3 were found more frequently in stage II/III/IV cases than in stage I cases. However, the significance of IQGAP3 ex- 
pression in GC remains unclear. Therefore, we next investigated the effect of IQGAP3 inhibition on sphere number and size on GC cells using siRNA.

First, we performed Western blot analysis of IQGAP3 in 4 GC cell lines. IQGAP3 protein expression was detected in MKN-45 and MKN-74 GC cells (Fig. 2a), so these 2 cell lines were selected for the following assays. Western blot analysis revealed that the expression of IQGAP3 in MKN-74 cells was substantially suppressed by treatment with siRNA1 and siRNA2 (Fig. 2b), while MTT assays showed that IQGAP3 siRNA1- and siRNA2transfected MKN-74 cells had significantly reduced cell growth relative to negative control siRNA-transfected cells (Fig. 2c). We also obtained similar results from an MTT assay performed 4 days after siRNA transfection of MKN-45 cells (data not shown). These results suggest that IQGAP3 is involved in GC cell growth.

The effect of IQGAP3 inhibition on sphere number and size was also examined in MKN-74 cells. Both the number (Fig. 2d) and size (Fig. 2e) of spheres formed by MKN-74 cells was significantly reduced in IQGAP3 siRNA1- and siRNA2-transfected cells compared with negative control siRNA-transfected cells. Similar results were obtained for spheres formed by MKN-45 cells (data not shown). These results suggest that IQGAP3 plays an important role in spheroid formation.

Effect of IQGAP3 Inhibition on Akt and Erk Pathways

Akt and Erk pathways are major signaling pathways that promote cancer cell proliferation [19], while IQGAP3 is known to interact with Erk1 and enhance its phosphorylation $[9,14]$. We therefore examined the effect of IQGAP3 inhibition on Akt and the Erk pathways in MKN-74 cells. Western blot analysis revealed that the levels of phosphorylated Akt and Erk1/2 were lower in IQGAP3 siRNA-transfected cells than in negative control siRNA-transfected cells (Fig. 2b). Similar results were observed in MKN-45 cells (data not shown).

\section{Discussion}

Previously, we identified several genes that were upregulated in spheroid body-forming GC cells compared with parental cells [6]. Among these, we reported the upregulation of KIFC1 and KIF11 proteins in GC cases [6, 7], and showed that both the number and size of spheres from GC cell lines were significantly reduced by knockdown of KIFC1 and KIF11. However, these 2 proteins are localized in the nucleus. In the present study, we focused on transmembrane proteins because the genes that encode them are potentially useful therapeutic targets or biomarkers for cancer diagnosis [8]. We found that IQGAP3 was upregulated in $21 \%$ of GC cases. A marginally significant difference was observed between IQGAP3 expression and T classification $(p=0.0783)$. Previously, it has been reported that IQGAP3 silencing in breast cancer cells repressed cell migration and invasion [20]. Thus, there is a possibility that IQGAP3 may stimulate GC cell invasion. IQGAP3 expression was statistically associated with $\mathrm{N}$ classification $(p=0.0132)$ and $\mathrm{M}$ classification $(p=0.0139)$. GC cases positive for IQGAP3 were found more frequently in stage II/III/IV cases than in stage I cases. Taken together, these results suggest that IQGAP3 plays an important role in the progression of GC. Furthermore, IQGAP3 expression was associated with survival, and served as an independent prognostic classifier of patients with GC. These results suggest that IQGAP3 immunostaining is a clinically useful method for the prediction of GC patient survival.

In the present study, MTT assays demonstrated that IQGAP3 knockdown inhibited cell growth. Although the underlying mechanisms remain unclear, we showed that the levels of phosphorylated Akt and Erk were lower in IQGAP3 siRNA-transfected GC cells than in negative control siRNA-transfected cells. IQGAP3 has previously been reported to interact with Erk1 and to enhance Erk1 phosphorylation following treatment with epidermal growth factor [9]. Because Erk signaling induces cell proliferation [19], it is likely to play an important role in promoting GC cell growth through interaction with IQGAP3. In contrast, the association between Akt signaling and IQGAP3 has not yet been examined, although the knockdown of IQGAP1 was shown to suppress the activation of Akt by human epidermal growth factor receptor 2. Additionally, the activation of Akt in mouse cardiac tissue induced by chronic pressure overload was not detected in IQGAP1-null mice [21]. Although it is conceivable that IQGAP3 also activates Akt signaling, further work is required to clarify this.

CSCs are defined as a unique subpopulation of cells that possess self-renewal and differentiation potential, and that are generally responsible for tumor initiation, invasion, metastasis, and chemo-resistance [22]. Thus, CSC characterization can improve our understanding of carcinogenesis and help explain the frequency of treatment failure for GC. In the present study, we showed that knockdown of IQGAP3 inhibited spheroid formation of GC cells. Previously, the Akt and Erk signaling pathways were shown to be essential for maintaining the pluripo- 
tency of stem cells $[23,24]$. Moreover, gastric spheroid cells are known to overexpress multiple stem cell markers, including Oct4, Sox2, Gli1, CD44, CD133, pAkt, and pErk [22]. Taken together, these results suggest that the phosphorylation of Akt and Erk by IQGAP3 is involved in spheroid formation by GC cells. However, CSCs are a minority population $(<5 \%)$ of cells [25], and we showed here that IQGAP3 is not a specific marker for gastric CSCs because the percentage of IQGAP3-stained GC cells ranged from 0 to $70 \%$.

The overexpression of IQGAP3 has been reported in several human cancers including hepatocellular carcinoma, lung cancer, pancreatic cancer, and breast cancer [ 9 , $20,26,27]$. IQGAP3 is located at $1 \mathrm{q} 21.3$, which is a hotspot for gene amplification in cancer [28]. DNA amplification at 1q21.3 has previously been reported in GC [29], so 1q21.3 amplification may contribute to the upregulation of IQGAP3. Future work should examine the association between 1q21.3 amplification and IQGAP3 expression.
In summary, we demonstrated that IQGAP3 is overexpressed in GC, and that IQGAP3 expression is an independent prognostic classifier of patients with GC. We also showed that knockdown of IQGAP3 inhibited cell growth and spheroid formation. Because IQGAP3 is a transmembrane protein, antibodies against IQGAP3 may constitute promising anticancer drugs.

\section{Acknowledgments}

This work was supported by Grants-in-Aid for Scientific Research (B-15H04713) and for Challenging Exploratory Research (26670175, 16K15247) from the Japan Society for the Promotion of Science, and by the Takeda Science Foundation. We thank Sarah Williams, PhD, from Edanz Group (www.edanzediting.com) for editing a draft of this manuscript.

\section{Disclosure Statement}

The authors declare no conflict of interest.

\section{References}

1 Oue N, Sentani K, Sakamoto N, Yasui W: Clinicopathologic and molecular characteristics of gastric cancer showing gastric and intestinal mucin phenotype. Cancer Sci 2015; 106:951-958.

2 Oue N, Naito Y, Hayashi T, Takigahira M, Kawano-Nagatsuma A, Sentani K, Sakamoto N, Zarni Oo H, Uraoka N, Yanagihara K, Ochiai A, Sasaki H, Yasui W: Signal peptidase complex 18, encoded by SEC11A, contributes to progression via TGF- $\alpha$ secretion in gastric cancer. Oncogene 2014;33:3918-3926.

3 Bessede E, Dubus P, Megraud F, Varon C: Helicobacter pylori infection and stem cells at the origin of gastric cancer. Oncogene 2015; 34:2547-2555.

4 Wakamatsu Y, Sakamoto N, Oo HZ, Naito Y, Uraoka N, Anami K, Sentani K, Oue N, Yasui $\mathrm{W}$ : Expression of cancer stem cell markers ALDH1, CD44 and CD133 in primary tumor and lymph node metastasis of gastric cancer. Pathol Int 2012;62:112-119.

5 Takaishi S, Okumura T, Wang TC: Gastric cancer stem cells. J Clin Oncol 2008;26:28762882.

6 Oue N, Mukai S, Imai T, Pham TT, Oshima T, Sentani K, Sakamoto N, Yoshida K, Yasui $\mathrm{W}$ : Induction of KIFC1 expression in gastric cancer spheroids. Oncol Rep 2016;36:349355.
7 Imai T, Oue N, Nishioka M, Mukai S, Oshima T, Sakamoto N, Sentani K, Matsusaki K, Yoshida K, Yasui W: Overexpression of KIF11 in gastric cancer with intestinal mucin phenotype. Pathobiology 2017;84:16-24.

8 Yasui W, Sentani K, Sakamoto N, Anami K, Naito Y, Oue N: Molecular pathology of gastric cancer: research and practice. Pathol Res Pract 2011;207:608-612.

9 Yang Y, Zhao W, Xu QW, Wang XS, Zhang Y, Zhang J: IQGAP3 promotes EGFR-ERK signaling and the growth and metastasis of lung cancer cells. PLoS One 2014;9:e97578.

10 Johnson M, Sharma M, Henderson BR: IQGAP1 regulation and roles in cancer. Cell Signal 2009;21:1471-1478.

11 Kim H, White CD, Sacks DB: IQGAP1 in microbial pathogenesis: targeting the actin cytoskeleton. FEBS Lett 2011;585:723-729.

12 Mataraza JM, Briggs MW, Li Z, Entwistle A, Ridley AJ, Sacks DB: IQGAP1 promotes cell motility and invasion. J Biol Chem 2003;278: 41237-41245.

13 Schmidt VA, Chiariello CS, Capilla E, Miller F, Bahou WF: Development of hepatocellular carcinoma in Iqgap2-deficient mice is IQGAP1 dependent. Mol Cell Biol 2008;28: 1489-1502.

14 Nojima H, Adachi M, Matsui T, Okawa K, Tsukita S, Tsukita S: IQGAP3 regulates cell proliferation through the Ras/ERK signalling cascade. Nat Cell Biol 2008;10:971-978.
15 Sobin LH, Gospodarowicz MK, Wittekind $\mathrm{CH}$ (eds): TNM Classification of Malignant Tumours, ed 7. New York, Wiley-Liss, 2009, pp 73-77.

16 Kondo T, Oue N, Yoshida K, Mitani Y, Naka $\mathrm{K}$, Nakayama $\mathrm{H}$, Yasui W: Expression of POT1 is associated with tumor stage and telomere length in gastric carcinoma. Cancer Res 2004;64:523-529.

17 Sakamoto N, Oue N, Sentani K, Anami K, Uraoka N, Naito Y, Oo HZ, Hinoi T, Ohdan H, Yanagihara K, Aoyagi K, Sasaki H, Yasui W: Liver-intestine cadherin induction by epidermal growth factor receptor is associated with intestinal differentiation of gastric cancer. Cancer Sci 2012;103:1744-1750.

18 Yasui W, Ayhan A, Kitadai Y, Nishimura K, Yokozaki $\mathrm{H}$, Ito $\mathrm{H}$, Tahara E: Increased expression of p34cdc2 and its kinase activity in human gastric and colonic carcinomas. Int J Cancer 1993;53:36-41.

19 Li S, Schmitz KR, Jeffrey PD, Wiltzius JJ, Kussie $P$, Ferguson KM: Structural basis for inhibition of the epidermal growth factor receptor by cetuximab. Cancer Cell 2005;7:301-311.

$20 \mathrm{Hu} \mathrm{G}, \mathrm{Xu} \mathrm{Y}$, Chen W, Wang J, Zhao C, Wang M: RNA interference of IQ motif containing GTPase-activating protein 3 (IQGAP3) inhibits cell proliferation and invasion in breast carcinoma cells. Oncol Res 2016;24:455-461. 
21 White CD, Erdemir HH, Sacks DB: IQGAP1 and its binding proteins control diverse biological functions. Cell Signal 2012;24:826834.

22 Zhang X, Hua R, Wang X, Huang M, Gan L, Wu Z, Zhang J, Wang H, Cheng Y, Li J, Guo W: Identification of stem-like cells and clinical significance of candidate stem cell markers in gastric cancer. Oncotarget 2016;7:98159831.

23 Moon SH, Kim DK, Cha Y, Jeon I, Song J, Park KS: PI3K/Akt and Stat3 signaling regulated by PTEN control of the cancer stem cell population, proliferation and senescence in a glioblastoma cell line. Int J Oncol 2013;42: 921-928.
24 Li J, Wang G, Wang C, Zhao Y, Zhang H, Tan Z, Song Z, Ding M, Deng H: MEK/ERK signaling contributes to the maintenance of human embryonic stem cell self-renewal. Differentiation 2007;75:299-307.

25 Al-Hajj M, Wicha MS, Benito-Hernandez A, Morrison SJ, Clarke MF: Prospective identification of tumorigenic breast cancer cells. Proc Natl Acad Sci USA 2003;100:3983-3988.

26 Qian EN, Han SY, Ding SZ, Lv X: Expression and diagnostic value of CCT3 and IQGAP3 in hepatocellular carcinoma. Cancer Cell Int 2016;16:55.
$27 \mathrm{Xu} \mathrm{W,} \mathrm{Xu} \mathrm{B,} \mathrm{Yao} \mathrm{Y,} \mathrm{Yu} \mathrm{X,} \mathrm{Cao} \mathrm{H,} \mathrm{Zhang} \mathrm{J,} \mathrm{Liu}$ J, Sheng H: Overexpression and biological function of IQGAP3 in human pancreatic cancer. Am J Transl Res 2016;8:5421-5432.

28 Wang S, Watanabe T, Noritake J, Fukata M, Yoshimura T, Itoh N, Harada T, Nakagawa M, Matsuura Y, Arimura N, Kaibuchi K: IQGAP3, a novel effector of Rac1 and Cdc42, regulates neurite outgrowth. J Cell Sci 2007; 120:567-577.

29 Vauhkonen H, Vauhkonen M, Sajantila A, Sipponen P, Knuutila S: DNA copy number aberrations in intestinal-type gastric cancer revealed by array-based comparative genomic hybridization. Cancer Genet Cytogenet 2006; 167:150-154. 